Canadian Oncology

Nursing Journal

Revue canadienne

de soins infirmiers

en oncologie

Volume 29, Issue 1 - Winter 2019

elSSN: 2368-8076 


\title{
Modification du point de vue des étudiants en sciences infirmières : répercussions d'une expérience d'observation préclinique dans un service d'oncologie externe
}

\author{
par Tracy L. Powell, Jordan Cooke et Alannah Brakke
}

\section{RÉSUMÉ}

Cette étude a examiné les répercussions d'une expérience d'observation en clinique externe d'oncologie sur les étudiants de première année en sciences infirmières. Cette occasion d'apprentissage par l'expérience a eu lieu avant tout cours officiel de pratique clinique. Une approche herméneutique de la phénoménologie a été utilisée pour découvrir le point de vue des étudiants en sciences infirmières sur le cancer avant l'obtention de leur permis d'exercice, ainsi que leur compréhension des rôles et des responsabilités des infirmières autorisées dans les soins liés au cancer, tant avant qu'après leur expérience. En tout, dix étudiants en sciences infirmières ont accepté de participer à l'étude. Les données ont été recueillies au moyen d'entrevues semi-structurées qui ont ensuite été analysées et interprétées pour comprendre la signification que l'expérience avait eue pour les étudiants. Deux thèmes principaux sont ressortis : les cercles de relations et les multiples facettes de l'oncologie ambulatoire. L'étude révèle que l'apprentissage par l'expérience sous la forme d'une expérience à faible risque, fondée uniquement sur l'observation, peut être une occasion pour les étudiants en préautorisation d'exercer d'acquérir de nouvelles perspectives sur les soins aux patients atteints de cancer et le rôle des infirmières dans les soins aux personnes et aux familles qui sont touchées par le cancer.

Mots-clés : étudiants en préautorisation d'exercer, expérience, cancer, apprentissage expérientiel, clinique externe, contexte ambulatoire

\section{AU SUJET DES AUTEURES \\ *Auteure-ressource : Tracy L. Powell, B.Sc.inf., inf. aut., M.Sc.inf., Professeure agrégée, École de sciences infirmières et pratique sage-femme, Faculté de santé, communauté et éducation, Mount Royal University, 4825 Mount Royal Gate SW, Calgary (Alberta) T3E 6K6}

Tél. : 403-390-8141 ; Téléc. : 403-440-6203 : Courriel : tlpowell@mtroyal.ca Jordan Cooke, B.Sc.inf., inf. aut., Médecine hospitalière interne, Traitement en clinique des toxicomanies, Alberta Health Services, Calgary (Alberta)

Alannah Brakke, B.Sc.inf., inf. aut., Réseau de soins de première ligne, West Springs Medical Inc., Calgary (Alberta)

DOI:10.5737/236880762914046

\section{INTRODUCTION}

T es professionnels de la santé interagissent avec des perL sonnes et des familles qui sont touchées par une gamme de maladies et de diagnostics. Comme le cancer touche un Canadien sur deux, les interactions avec ce groupe de personnes se produisent souvent dans tous les milieux de soins de santé. Par conséquent, il est important d'avoir des connaissances et une compréhension de la complexité de cette population afin de fournir des soins sûrs, éthiques et axés sur le patient (Comité directeur des statistiques de la Société canadienne du cancer, 2017).

Même chez les professionnels de la santé, la peur et les idées préconçues au sujet du cancer sont courantes, peu importe le type, le traitement ou le résultat (Box et Anderson, 1997; Dickinson, Clark et Sque, 2008; Cunningham, Copp, Collins et Bater, 2006; Komprood, 2013; Miller, Kearney et Smith, 2000). La littérature indique qu'être exposé à des personnes atteintes de différents types de cancer et aux symptômes associés, ou à différents milieux cliniques et domaines de traitement du cancer, ces points de vue des professionnels de la santé peuvent être modérés (Miller et al., 2000; Cunningham et al., 2006). Il est important de reconnaître qu'il existe des différences dans l'expérience, la présentation et le pronostic des patients atteints de cancer, en particulier pour les étudiants de premier cycle en sciences de la santé, tout comme il est important de faciliter l'acquisition de ces connaissances et d'avoir le temps de réfléchir à ces expériences pour les étudiants qui se préparent à entreprendre des stages cliniques où ils rencontreront des personnes et des familles touchées par le cancer (Cunningham et al., 2006; Ifanti, Iconomou, Viha et Kalofonos, 2009; Kav, Citak, Akman et Erdemir, 2013).

\section{CONTEXTE}

De 2007 à 2017, les étudiants de première année du baccalauréat en sciences infirmières (B.Sc.inf.) de la Mount Royal University's (MRU) ont eu l'occasion d'observer une infirmière autorisée en pratique avant de commencer leurs cours cliniques. Le but général était de mieux comprendre les rôles, les responsabilités et les domaines de pratique d'une infirmière autorisée. On s'attendait également à ce que chaque étudiant en sciences infirmières recueille des renseignements généraux sur les patients et les familles, sur le contexte et sur la façon dont les concepts de base appris dans ses cours théoriques en sciences infirmières se révélaient dans la pratique. 
Il était attendu des étudiants que cette expérience préclinique soit purement axée sur l'observation et qu'aucun soin direct ne puisse être fourni, peu importe le milieu, la tâche, la compétence ou l'expérience antérieure de l'étudiant (autres études ou expérience en tant qu'infirmière auxiliaire autorisée). Les étudiants étaient toutefois encouragés à communiquer et à poser des questions aux infirmières autorisées, aux patients, aux familles et aux professionnels de la santé. Les infirmières autorisées étaient informées de cette attente lorsqu'elles se portaient volontaires pour que les étudiants les accompagnent. Afin de commencer la socialisation dans leur rôle, on s'attendait aussi à ce que les étudiants respectent les exigences du code vestimentaire du programme de sciences infirmières. L'expérience d'observation de chaque étudiant a duré de trois à quatre heures, ce qui reflète le niveau de soutien des établissements de soins de santé.

Les infirmières autorisées bénévoles provenaient d'une variété d'unités et de milieux auxquels les étudiants avaient la possibilité d'avoir accès en observation. Sur la cohorte de 100 à 125 étudiants en sciences infirmières présents chaque trimestre, entre 20 et 35 avaient l'occasion de vivre une expérience avec une infirmière autorisée dans une clinique externe d'oncologie. Étant donné que cette activité a lieu avant tout stage clinique, la question s'est posée à savoir si cette occasion unique d'apprentissage par l'expérience favoriserait la pensée critique et la réflexion chez les étudiants. De plus, l'oncologie et les soins aux personnes atteintes de cancer présentaient un intérêt particulier étant donné que la documentation suggère que les paradigmes d'apprentissage qui exposent les étudiants à différentes dimensions du cancer peuvent contribuer à déloger les fausses idées courantes sur les diagnostics, les symptômes, les traitements et l'effet des soins prodigués aux patients (Cunningham et al., 2006; Komprood, 2013). Les enseignants se sont demandé si ces expériences avaient un impact sur les croyances et les attitudes des étudiants à l'égard du cancer, ainsi que sur leurs suppositions au sujet de leur rôle d'infirmière autorisée (Cunningham et al., 2006; Komprood, 2013).

\section{REVUE DE LA LITTÉRATURE}

Préparer les étudiants à l'entrée en pratique peut s'avérer difficile compte tenu des exigences concurrentes de préparation théorique versus pratique, particulièrement avec l'examen d'admission du National Council Licensure Examination Registered Nurses (NCLEX-RN) qui plane (McGillis Hall, Lalonde, Kashin, Yoo et Moran, 2018; Salfi et Carbol, 2017; Sellman, 2016). Les enseignants en sciences infirmières sont bien conscients de la nécessité de transmettre les concepts d'une manière évocatrice afin que le lien entre théorie et pratique se fasse (Adamski, Parsons et Hooper, 2009; Amir, Jelas et Rahman, 2011; Brennan et McSherry, 2007; Cano-Garcia et Hughes, 2000; Davidson, 2004; El Hussein, Salyers et Osuji, 2016; Gidman, 2013; Kolb, 1984; Özbas, 2013; Roberts, 2010). Favoriser les possibilités d'apprentissage par l'expérience grâce à des activités intégrées centrées sur l'apprenant est une approche pratique que des professions comme les soins infirmiers ont mise au point pour atteindre cet objectif
(Ironside, McNelis et Ebright, 2014; Wilson et al., 2009). On espère par ces techniques stimuler l'intégration des connaissances, la pensée critique et la réflexion.

Lapprentissage clinique (ou les stages cliniques) est une méthode d'apprentissage par l'expérience des plus utilisées dans nos professions. Ces occasions sont bénéfiques, mais auraient aussi des répercussions négatives sur les étudiants en raison des attentes en matière d'évaluation, de la peur des étudiants de causer du tort, de leur sentiment de manquer de connaissances et de la vulnérabilité connue des patients (Admi, 1997; Cooke, 1996; Henderson, Cooke, Creedy et Walker, 2012; Ironside, McNelis et Ebright, 2014; Suliman et Halabi, 2007; Murphy, 2004; Tanner, 2006). Or, les associations négatives sont courantes et les patients présentant une affection aiguë et complexe sont souvent au centre des soins hospitaliers en oncologie, un contexte clinique qui fait en sorte que les avantages connexes de la pratique clinique pour les étudiants en sciences infirmières sont encore plus susceptibles d'être altérés (Box et Anderson, 1997; Charalambous et Kaite, 2013; Nielsen, Noone, Voss et Mathews, 2013).

La simulation clinique haute fidélité (SCHF) constitue également une technique efficace utilisée pour favoriser l'apprentissage dans la formation des professionnels de la santé (Corvetto et Taekman, 2013; Paige, Arora, Fernando et Seymour, 2015). Le compte rendu qui suit obligatoirement la simulation, une caractéristique distinctive de la SCHF, est un élément clé pour améliorer l'apprentissage des étudiants (Janzen et al., 2016; Meakim et al., 2013; Wotton, Davis, Button et Kelton, 2010). Une autre technique d'apprentissage par l'expérience qui est moins reconnue dans la littérature est celle des expériences à faible risque fondées uniquement sur l'observation. Malgré sa faible présence dans la littérature, on signale cette méthode comme permettant de réduire certains obstacles à l'apprentissage et de susciter une réflexion approfondie sur les attitudes et perceptions (Admi, 1997; Mazerolle, Bowman et Benes, 2015; Meakim et al., 2013; Wong et Lee, 2000). Les possibilités d'apprentissage expérientiel par l'observation dans les programmes de sciences infirmières avec préautorisation d'exercer n'ont pas encore fait l'objet de recherches.

La prévalence du cancer est un incitatif à bien préparer les étudiants en sciences infirmières pour leur donner confiance en eux quand viendra le temps de prendre soin des personnes et familles touchées par cette maladie. Les étudiants en sciences infirmières ne se sentent généralement pas prêts à soigner les personnes chez qui on a diagnostiqué un cancer (Charalambous et Kaite, 2013; Frost, Brueggen et Mangan, 1997; Miller et al., 2000; Mohan, Wilkes, Ogunsiji et Walker, 2005; O'Connor et Fitzsimmons, 2005; Wyatt, 2007; Yildiz et Akansel, 2011), ce qui exacerbe leur sentiment d'anxiété, de stress et d'incompétence (Admi, 1997; Andrew, McGuiness, Reid et Corcoran, 2009; Cunningham et al., 2006; Miller et al., 2000; Sanford, Townsend-Rocchiccioli, Quiett et Trimm, 2011; Yildiz et Akansel, 2011). Certaines recherches ont montré que des expériences cliniques de soutien spécifique en oncologie peuvent accroitre la confiance et la compétence des étudiants (Mohan et al., 2005; Sanford et al., 2011). Offrir ces possibilités 
d'apprentissage dans divers milieux oncologiques a également été indiqué comme un moyen de modifier les idées fausses courantes sur le cancer, particulièrement en ce qui concerne la morbidité et la mortalité des patients (Komprood, 2013; Sanford et al., 2011).

Même si certaines études ont mis l'accent sur les perceptions du cancer et la prestation de soins aux patients atteints de cancer, peu de documentation est publiée sur les attitudes des étudiants en sciences infirmières avant, pendant ou après leur exposition à un milieu oncologique (Charalambous et Kaite, 2013). Les soins actifs continuent d'être le milieu courant où les étudiants en sciences infirmières sont exposés à des patients en oncologie. Les recherches sur l'exposition à l'extérieur de ce milieu sont rares (Cunningham et al., 2006; KingOkoye et Arber, 2014; Yildiz et Akansel, 2011). La recherche est limitée en ce qui concerne l'expérience des étudiants en sciences infirmières avant l'obtention de leur permis d'exercer dans différents types de milieux oncologiques, comme les soins ambulatoires, ainsi que l'impact de ces occasions uniques de stage clinique sur les croyances et les attitudes à l'égard des patients atteints de cancer et des patients en oncologie (Coyne et Needham, 2012).

\section{QUESTION DE RECHERCHE}

La présente étude visait à analyser l'expérience des étudiants en sciences infirmières ayant participé à une expérience d'observation par l'entremise des services de santé de l'Alberta au Tom Baker Cancer Centre de Calgary, en Alberta. On voulait s'intéresser particulièrement au sens de l'expérience pour les étudiants ainsi qu'à leurs perceptions et leurs croyances au sujet du cancer. La question de recherche a donc été formulée ainsi : Quelles expériences vivent les étudiants de première année en sciences infirmières qui profitent d'une occasion d'apprentissage expérientiel dans un contexte d'oncologie ambulatoire?

\section{MÉTHODOLOGIE}

Ce projet qualitatif a été guidé par une approche herméneutique de la phénoménologie (Guenther, 2014; van Manen, 1990). L'une des caractéristiques particulières de cette étude résidait dans le fait que la chercheuse principale jouait le double rôle de professeur (pour une section du cours à laquelle l'observation était associée) et de chercheur pour le projet. Par conséquent, un assistant de recherche s'est occupé du recrutement, de la distribution des formulaires de consentement et de la collecte de données afin de réduire autant que possible les conflits d'intérêts potentiels. L'approbation éthique de recherche avec des sujets humains a été obtenue avant le début de l'étude.

Avant la collecte des données, l'assistant de recherche a entrepris de dégager des pensées en cernant les idées préconçues qui circulaient sur les expériences des participants dans une clinique externe d'oncologie afin d'assurer la validité de l'étude. Nous avons recueilli les données au moyen d'entrevues individuelles semi-structurées en personne afin de mieux saisir la signification de l'expérience relatée par les participants (Guenther, 2014). Les données démographiques n'ont pas été recueillies dans le cadre de cette étude. Un total de dix étudiants de première année en sciences infirmières ayant participé à l'expérience d'observation à la clinique externe du cancer (la moitié à la session d'automne 2015 et l'autre moitié à l'hiver 2016) ont accepté de participer à l'étude. Il n'y avait pas de différence dans les connaissances ou l'expérience des participants en raison du programme d'études - tous avaient les mêmes préalables et cours concomitants. Les secteurs de la clinique externe d'oncologie que les étudiants pouvaient éventuellement observer étaient la greffe de moelle osseuse, les traitements de chimiothérapie, les essais cliniques, les cliniques externes et la radiothérapie. Chaque entrevue avec les participants a été enregistrée sur appareil numérique puis retranscrite mot pour mot. Au moment de la transcription, tous les renseignements permettant d'identifier les participants ont été supprimés afin de préserver leur confidentialité.

L’approche de Van Manen (1990) de phénoménologie herméneutique a été utilisée pour effectuer l'analyse et l'interprétation des données et faire ressortir des thèmes et des énoncés connexes dans les descriptions. Une fois les données préparées et la session d'hiver 2016 terminée, la chercheuse principale a participé à l'exercice visant à dégager les pensées avant de passer à la lecture des transcriptions. Pour obtenir une première impression, chaque transcription a fait l'objet d'une lecture individuelle. De multiples relectures de l'ensemble des transcriptions ont suivi afin de déterminer les thèmes liés aux expériences d'observation. Les principaux énoncés de chaque fichier de données ont été soulignés, les thèmes récurrents ont été indiqués et, au cours des lectures subséquentes, d'autres thèmes et énoncés ont été notés et soulignés (van Manen, 1990). Tous les énoncés ayant une signification similaire ont été regroupés en thèmes qui ont ensuite fait l'objet d'une interprétation plus poussée (Dhotre, Adams, Hebert, Bottai et Heiney, 2016). Ces thèmes essentiels ont ensuite été décrits à l'aide d'une explication ou d'une citation du participant (van Manen, 1990).

\section{CONSTATS}

Deux grands thèmes sont ressortis des données : a) les cercles de relations; b) les multiples facettes de l'oncologie ambulatoire. Les idées essentielles qui ont été ressorties en rapport avec les cercles de relations sont les suivantes : « équipes de soins axées sur la collaboration », « comme une famille » et «le juste milieu ». Les idées essentielles qui sont ressorties du constat concernant les multiples facettes de l'oncologie ambulatoire sont les suivantes : «le cancer est quelque chose de personnel », « le côté calme des soins » et « l'éducation comme forme de soin ».

\section{Thème 1 : Les cercles de relations}

Ce thème reflétait le point de vue des participants sur les relations qui font partie du contexte de l'oncologie ambulatoire. Plutôt que de considérer ces collaborations comme des dyades, les participants ont que les espaces et les interactions s'entrelaçaient, se chevauchaient et s'appuyaient sur les relations. 


\section{Idée 1.1}

L'idée des « équipes de soins axées sur la collaboration » représentait les relations entre les professionnels de la santé et les patients, telles qu'indiquées par les participants. Le rôle des professionnels de la santé, des patients et des infirmières autorisées à la clinique d'oncologie n'était pas comme ils le croyaient. Les participants s'attendaient à une « énorme hiérarchie » entre les oncologues et les autres membres de l'équipe, supposant que le paternalisme serait évident, d'après les impressions préconçues qu'ils avaient des équipes de soins de santé, auxquelles ils n'avaient en fait jamais été exposés. Comme l'a décrit un participant :

Toute cette conversation sur [...] qui avait un problème avec les somnifères qu'elle prenait... Le pharmacien l'avait appelée pour lui exposer le problème, puis l'infirmière autorisée lui avait dit quoi faire entre-temps, et là elle attendait de voir son médecin de famille. C'était donc intéressant d'entendre les trois [...] ...de voir comment les trois interventions se sont recoupées. Comment [...] ont travaillé ensemble pour faire en sorte que les choses fonctionnent. (P-001)

\section{Idée 1.2}

Le thème « comme une famille » faisait référence au niveau et au type de relations que les infirmières autorisées, les patients et leurs familles entretenaient. Les participants ont indiqué que la chaleur et la familiarité étaient évidentes dans leurs observations des relations, qui s'écartaient des relations distantes et froides auxquelles ils s'attendaient. La manière de communiquer et les liens que beaucoup d'infirmières autorisées entretenaient avec leurs patients étaient « professionnels » et « respectueux », mais les participants ont dit que ce n'était pas ce qu'ils avaient interprété dans leurs manuels ou leurs cours jusqu'à maintenant dans le cadre du programme de sciences infirmières. Comme l'a mentionné un participant:

J'ai été vraiment surpris de voir à quel point les infirmières étaient attachées émotionnellement aux patients. Parce qu'une patiente devait partir la semaine d'après, et l'infirmière que j'accompagnais, elle était vraiment bouleversée parce que la semaine d'après, elle ne serait pas en ville, et elle est devenue très émotive avec le patient. Et le patient est devenu émotif aussi. Je n'avais jamais réalisé à quel point ça pouvait être émotionnel. Parce qu'ils se voient depuis environ six mois pour [...] et se sont vraiment attachés l'un à l'autre. (P-003)

L'ouverture d'esprit et le soutien dont font preuve les professionnels de la santé les uns envers les autres ont également été soulignés :

Et cétait intéressant de voir, genre, à quel point tout le monde est proche les uns des autres. Toutes les infirmières, les résidents et les pharmaciens sont très proches. Je veux dire, ils se connaissent vraiment bien. Pas juste sur le plan professionnel, ils connaissaient leur vie, leur vie personnelle. (P-003)

\section{Idée 1.3}

Le thème « le juste milieu » touche la compréhension du cancer chez les participants et leur perception de ce que l'on attendait d'eux et dans le contexte de l'oncologie. Les participants s'attendaient à ce que le milieu soit un « endroit triste », mais au fur et à mesure qu'ils interagissaient avec les infirmières autorisées, les patients, les familles et les autres membres de l'équipe de soins de santé, ils ont compris que les patients et les infirmières étaient empathiques envers les expériences des uns et des autres et leur entourage.

J'ai réalisé à quel point cela peut être réel avec le patient; comment cela affecte honnêtement sa vie. Parce que les patients, bien qu'ils soient vraiment tristes, ils sont aussi... Ils ne veulent pas que toute leur vie soit centrée sur eux. Ils sont humains; ils veulent écouter les problèmes des autres. (P-003)

Les participants ont compris que le diagnostic de cancer pouvait toucher des personnes de tous milieux et de toutes expériences de vie, et qu'une personne n'est pas « uniquement un patient atteint de cancer ». L'horizon des participants s'est élargi à mesure qu'ils ont pris conscience que certains patients atteints de cancer devaient - et dans certains cas même voulaient et désiraient - assumer d'autres rôles et responsabilités, que leur vie ne se réduisait pas au cancer:

....Ça m'a surpris de voir à quel point certains patients étaient contents. Il $y$ avait un type en costume d'affaires. Il avait son ordinateur portable et travaillait pendant sa thérapie systémique. (P-004)

La nature dynamique du milieu et la capacité de l'infirmière de répondre aux nombreuses demandes qui lui sont adressées ont également émergé :

J'ai donc été surpris de voir à quel point les infirmières étaient... directes, mais aussi, euh, douces... De plus, il $y$ avait ces cinq patients dans la salle d'attente... Mais elles prennent quand même le temps de répondre à toutes les questions. (P-010)

\section{Thème 2 : Les multiples facettes de l'oncologie ambulatoire}

Ce thème est apparu en relation avec le contexte des croyances préconçues sur le diagnostic de cancer et le milieu clinique. Les participants ont réalisé qu'il était important de reconnaître le contexte dans lequel se trouvaient les personnes et familles touchées par le cancer. Ils ont également pris conscience de la nature dynamique de la maladie et de l'impact du milieu ambulatoire en contexte de cancer.

\section{Idée 2.1}

L'idée que «le cancer est quelque chose de personnel » décrit la compréhension acquise du fait que chaque personne chez qui on diagnostique un cancer est unique et individuelle. L'attitude des patients atteints de cancer et de leur famille ainsi que la façon dont l'environnement clinique lui-même exposait les situations individuelles étaient des plus révélatrices. Comme l'a dit un participant:

Chaque patient qui arrivait était différent et avait un... Ils avaient tous... Ils semblaient tous avoir des attitudes réellement positives, ce que la plupart des gens ne s'attendraient pas à voir dans une unité d'oncologie. (P-001)

De nombreux participants ont également décrit mieux comprendre le cancer en tant que diagnostic et les différentes évaluations objectives des patients par rapport aux symptômes et 
à la tolérance au traitement. Pour certains participants, leur vision du cancer s'est aussi transformée :

... Et ils n'avaient pas l'air vraiment malades. Je suppose que oui. Ils semblaient très... Je sais ce que nous apprenons, que la santé est abstraite, mais ils m’ont semblé très sains, décontractés et normaux. Et ça m'a fait réaliser que n'importe qui peut avoir n'importe quelle maladie. Ça m'a ouvert l'esprit. (P-003)

\section{Idée 2.2}

Le thème " côté calme des soins » reflète le contexte et les attentes des participants à l'égard d'un environnement clinique intense où les activités se déroulent à un rythme rapide. La plupart des participants s'attendaient à trouver des patients cancéreux «traumatisés » et éprouvant des nausées, des douleurs et de la détresse extrêmes; ils ont compris que dans un contexte d'oncologie ambulatoire, les soins prenaient un autre visage :

Quand les gens ont eu une réaction, mais qu'ils s'y attendaient. Ils savent quels médicaments causent quelles réactions, alors en recevant ce médicament, ils savent quoi surveiller. Et ils sont rapides. Comme cette dame quand elle a commencé sa réaction et a rougi d'un seul coup, mais à peu près 30 à 45 secondes plus tard je dirais, elle était revenue à la normale. (P-001).

Ce participant avait beaucoup de respect pour la façon dont les infirmières traitaient le patient et pouvaient reprendre leurs responsabilités une fois que le patient avait répondu au traitement. De façon constante, les participants ont été surpris de l'ambiance et du sentiment général de paix qui régnait dans le milieu.

\section{Idée 2.3}

Le thème «l'éducation comme forme de soin » est apparu dans les données à mesure que les participants comprenaient les rôles et responsabilités des infirmières à la clinique d'oncologie, ainsi que les domaines dans lesquels elles travaillaient. Il s'est avéré, grâce à l'éducation, que les infirmières fournissaient un type de soins essentiels aux patients et à leurs familles. Les participants ont indiqué que sans cette éducation, l'expérience et les résultats pour les patients auraient été grandement modifiés. Comme l'a signalé un participant :

J'ai en quelque sorte pu voir l'infirmière dans son rôle d'enseignante. Elle a montré comment faire des piqûres et comment vraiment aider le patient et sa femme à travers tout ça... C'était vraiment très intéressant. Elle (linfirmière) a été très positive tout le long. (P-009)

Certains participants ont parlé de la façon dont l'éducation était dispensée aux patients. Comme l'a dit un participant, cétait « très thérapeutique, très direct, mais sans aucun manque de respect, et en respectant toujours leur situation. (P-010).

\section{DISCUSSION}

En tant qu'occasion d'apprentissage expérientiel, l'observation dans une clinique externe d'oncologie a eu une influence sur la compréhension globale des rôles, des responsabilités et des relations des infirmières. Cela a également permis aux étudiants de mieux comprendre les patients atteints de cancer et leurs besoins en matière de soins. Bien que certains étudiants en sciences infirmières qui ont fréquenté le milieu ambulatoire auraient préféré au départ vivre leur expérience d'observation préclinique dans un milieu autre que l'oncologie, tous étaient unanimes après pour dire que leur perspective sur le cancer, les soins du cancer et l'image des patients en oncologie avait changé pour le mieux.

Étant donné que l'expérience d'observation s'est déroulée avant tout stage clinique et que, pour plusieurs participants, il s'agissait d'une première exposition à un milieu clinique de ce genre, certains des thèmes qui sont ressortis étaient peut-être le résultat de connaissances globales limitées de la part des étudiants. Toutefois, la documentation révèle que les programmes de sciences infirmières du premier cycle manquent généralement de contenu sur le cancer ou que le contenu est éparpillé dans le programme d'études, de sorte qu'il n'y a que peu à en retirer (Box et Anderson 1997; Cheung, Fishman et Verma, 2009; Komprood, 2013; Miller et al., 2000; Mohan et al., 2005; O'Connor et Fitzsimmons 2005; Sanford et al., 2011). Par conséquent, il est peu probable que les connaissances acquises par les étudiants participants à cette étude aient été significativement différentes même si l'expérience s'était produite plus tard dans leur formation en sciences infirmières (King-Okoye et Arber, 2014; Yildiz et Akansel, 2011).

Les étudiants en sciences infirmières de cette étude ont été en mesure d'exprimer un certain lien entre la théorie apprise au cours de la première année de leur diplôme et les aspects observés pendant l'exposition clinique, de même que les personnes avec lesquelles ils ont interagi. En particulier, les concepts de collaboration, de santé et de maladie, et de soins ont été remarqués. Faire des liens entre la théorie et la pratique est essentiel tant pour les étudiants que pour les infirmières en pratique (Edwards, Anstey, Kelly, Ballie et Hopkins, 2017). Transmettre des concepts abstraits est souvent un défi dans les cours théoriques, particulièrement lorsqu'il y a peu ou pas de contexte clinique (El Hussein, Salyers et Osuji, 2016). En utilisant cette expérience d'observation à faible risque en conjonction avec un cours théorique pour les étudiants en sciences infirmières avant l'obtention de leur permis d'exercice, les participants à cette étude ont indiqué une acquisition de connaissances liées à l'oncologie et une modification de leurs croyances et idées fausses concernant l'oncologie, les soins du cancer et les rôles des infirmières autorisées.

\section{CONCLUSION}

Cette recherche était axée sur le recours à l'apprentissage expérientiel sous forme d'observation préclinique pour permettre aux étudiants d'acquérir des connaissances par un autoapprentissage fondé sur la pratique (Barr et Tagg, 1995; Crookes, Crookes et Walsh, 2013). Cette recherche a mesuré les répercussions de l'apprentissage expérientiel à faible risque sur un groupe d'étudiants en sciences infirmières avant l'obtention du permis d'exercice et mis en lumière l'expérience acquise par ces étudiants en clinique externe d'oncologie. Les constats tirés apportent de l'eau au moulin lorsque vient 
le temps pour les pédagogues et leaders des soins infirmiers en oncologie de trouver comment intégrer les connaissances en oncologie et en soins du cancer au programme d'études. Ils éclairent aussi l'influence de techniques d'apprentissage novatrices dans les domaines de pratique par rapport aux idées préconçues. Il pourrait donc en résulter des avantages

\section{RÉFÉRENCES}

Adamski, M., Parsons, V., \& Hooper, C. (2009). Internalizing the concept of caring: An examination of student perception when nurses share their stories. Nursing Education Research, 30(6), 358-361.

Admi, H. (1997). Nursing students: stress during the initial clinical experience. Journal of Nursing Education, 36, 323-327.

Amir, R., Jelas, Z.M., \& Rahman, S. (2011). Learning styles of university students: Implications for teaching and learning. World Applied Sciences Journal, 14, 22-26.

Andrew, N., McGuiness, C., Reid, G., \& Corcoran, T. (2009). Greater than the sum of its parts: Transition into the first year of undergraduate nursing. Nursing Education in Practice, 9, 13-21. doi:10.1016/j.nepr.2008.03.009.

Barr, R., \& Tagg, J. (1995). From teaching to learning: A new paradigm for undergraduate education. Change, 27(6), 13-25.

Box, V., \& Anderson, Y. (1997). Cancer beliefs, attitudes and preventative behaviours of nurses working in the community. European Journal of Cancer Care, 6, 192-208.

Brennan, G., \& McSherry, R. (2007). Exploring the transition and professional socialization from health care assistant to student nurse. Nurse Education in Practice, 7, 206-214. doi:10:1016/j. nepr.2006.08.006

Canadian Cancer Society's Advisory Committee on Cancer Statistics. (2017). Canadian cancer statistics 2017. Toronto, ON: Canadian Cancer Society 2017.

Charalambous, A., \& Kaite, C. (2013). Undergraduate nursing students caring for cancer patients: Hermeneutic phenomenological insights of their experiences. BMC Health Services Research, 13(63), $1-13$.

Cheung, W.Y., Fishman, P.N., \& Verma, S. (2009). Oncology education in Canadian undergraduate and postgraduate training programs. Journal of Cancer Education, 24(4), 284-290. doi:10.1080/08858190902973143

Cano-Garcia, F., \& Hughes, E.H. (2000). Learning and thinking styles: An analysis of their interrelationship and influence on academic achievement. Educational Psychology, 20, 413-430. doi:10.1080/713663755

Cooke, M. (1996). Nursing students' perceptions of difficult or challenging clinical situations. Journal of Advanced Nursing, 24(6), 1281-1287.

Corvetto, M.A., \& Taekman, J.M. (2013). To die or not to die? A review of simulated death. Simulation in Healthcare, 8(1), 8-12. doi:10.1097/SIH0b013e3182689aff

Coyne, E., \& Needham, J. (2012) Undergraduate nursing students' placement in speciality clinical areas: Understanding the concerns of the student and registered nurse. Contemporary Nurse, 42(1), 97-104. doi:10.5172/conu.2012.42.1.97

Crookes, K., Crookes, P.A., \& Walsh, K. (2013). Meaningful and engaging teaching techniques for student nurses: A literature review. Nursing Education in Practice, 13, 239-243. doi:10.1016/j. nepr.2013.04.008

Cunningham, S.M., Copp, G., Collins, B., \& Bater, M. (2006). Preregistration nursing students' experience of caring for cancer potentiels pour les étudiants, les patients, les familles et le système de santé.

\section{REMERCIEMENTS}

Nous remercions l'ACIO/CANO et le comité exécutif de l'ACIO/ CANO pour la bourse de recherche de 2015 qui a soutenu ce projet.

patients. European Journal of Oncology Nursing, 10, 59-67. doi:10.1016/j.ejon.2005.05.004.

Davidson, M.R. (2004). A phenomenological evaluation: Using storytelling as a primary teaching method. Nurse Education in Practice, 4(3), 184-189. doi:10.1016/S1471-5953(03)00043-X.

Dhotre, K.B., Adams, S.A., Hebert, J.R., Bottai, M., \& Heiney, S.P. (2016). Oncology nurses' experiences with patients who chose to discontinue cancer chemotherapy. Oncology Nursing Forum, 43(5), 617-623. doi:10.1188/16.ONF.617-623

Dickinson, G.E., Clark, D., \& Sque, M. (2008). Palliative care and end of life issues in UK pre-registration, undergraduate nursing programmes. Nurse Education Today, 28(2), 163-170. doi:10.1016/j. nedt.2007.03.008

Edwards, D., Anstey, S., Kelly, D., Ballie, J., \& Hopkins, J. (2017). What is important for student nurses to know about cancer treatment and care: A qualitative study of student nurses' and stakeholder perspectives. Journal of Clinical Nursing, 26(13-14), 2045-2054. doi:10.1111/jocn.13616

El Hussein, M.T., Salyers, V., \& Osuji, J. (2016). Use of visual narrative illustrations to teach pathophysiology concepts to nursing students. Journal of Nursing Education, 55(2), 109-112. doi:10.3928/01484834-20160114-10

Frost, M.H., Brueggen, C., \& Mangan, M. (1997). Intervening with the psychosocial needs of patients and families: Perceived importance and skill level. Cancer Nursing, 20, 350-358.

Gidman, J. (2013). Listening to stories: Valuing knowledge from patient experience. Nursing Education in Practice, 13(3), 192-196. doi:10.1016/j.nepr.2012.09.006

Guenther, J.L. (2014). A phenomenological approach to describe the lived experience of ovarian cancer. Journal of Obstetric, Gynecological and Neonatal Nursing, 43(S1), S81. doi:10/1111/1552-6909.12438

Henderson, A., Cooke, M., Creedy, D., \& Walker, R. (2012). Nursing students' perceptions of learning in practice environments: A review. Nursing Education, 32(3), 299-302. doi:10.1016/j. nedt.2011.03.010

Ifanti, A.A., Iconomou, G., Viha, A., \& Kalofonos, H.P. (2009). Undergraduate nursing students' view on oncology: A case study in Greece. Journal of Balkan Union of Oncology, 14, 689-698.

Ironside, P.M., McNelis, A.M., \& Ebright, P. (2014). Clinical education in nursing: Rethinking learning in practice settings. Nursing Outlook, 62(3), 185-191. doi:10.1016/j.outlook.2013.12.004

Janzen, K.J., Jeske, S., MacLean, H., Harvey, G., Nickle, P., Norenna, L., Holtby, M., \& McLellan, H. (2016). Handling strong emotions before, during, and after simulated clinical experiences. Clinical Simulation in Nursing, 12(2), 37-43. doi:10.1016/j.ecns.2015.12.004

Kav S., Citak E.A., Akman A., \& Erdemir F. (2013). Nursing students' perceptions towards cancer and caring for cancer patients in Turkey. Nurse Education in Practice, 13(1), 4-10. doi:10.1016/j. nepr.2012.05.010

King-Okoye, M., \& Arber, A. (2014). 'It stays with me': The experiences of second- and third-year student nurses when caring for patients with cancer. European Journal of Cancer Care, 23(4), 441-449. doi:10.1111/ecc.12139 
Kolb, D.A. (1984). Experiential learning. Englewood Cliffs: PrenticeHall, Inc.

Komprood, S.R. (2013). Nursing student attitudes toward oncology nursing: An evidence-based literature review. Clinical Journal of Oncolog $\gamma$ Nursing, 17(1), E21-28.

Mazerolle, S.M., Bowman, T.G., \& Benes, S.S. (2015). Reflective observation in the clinical education setting: A way to promote learning. Journal of Athletic Training, 10(1), 32-38. doi:10.4085/100132

McGillis Hall, L., Lalonde, M., Kashin, J., Yoo, C., \& Moran, J. (2018). Changing nursing licensing examinations: Media analysis and implications of the Canadian experience. International Nursing Review, 65(1), 13-23. doi:10.1111/inr.12367

Meakim, C., Boese, T., Decker, S., Franklin, A.E., Gloe, D., Lioce, L., \& Borum, J.C. (2013). Standards of best practice: Standard I terminology. Clinical Simulation in Nursing, 9(6S), S3-S11.

Miller, M., Kearney, N., \& Smith, K. (2000). Measurement of cancer attitudes: A review. European Journal of Oncology Nursing, 4, 233245. doi:10.1054/ejon.2000.0109

Mohan, S., Wilkes, L.M., Ogunsiji, O., \& Walker, A. (2005). Caring for patients with cancer in non-specialist wards: The nurse experience. European Journal of Cancer Care, 14, 256-263.

Murphy, J.I. (2004). Using focused reflection and articulation to promote clinical reasoning: An evidence-based teaching strategy. Nursing Education Perspectives, 25, 226-231.

Nielsen, A.E., Noone, J., Voss, H., \& Mathews, L.R. (2013). Preparing nursing students for the future: An innovative approach to clinical education. Nurse Education in Practice, 13(4), 301-309. doi:10.1016/j. nepr.2013.03.015

O'Connor, S.J., \& Fitzsimmons, D. (2005). Embedding cancer care within pre-registrations nurse education programmes: Policy, practice and opportunities for change. European Journal of Oncology Nursing, 9, 341-350. doi.10.1016/j.ejon.2005.02.002

Özbas, S. (2013). The investigation of the learning styles of university students. The Online Journal of New Horizons in Education, 3(1), 53-64.

Paige, J.T., Arora, S., Fernandez, G., \& Seymour, N. (2015). Debriefing 101: Training faculty to promote learning in simulation-based training. The American Journal of Surgery, 209(1), 126-131. doi:10.1016.j.amjsurg.2014.05.034
Roberts, D. (2010). Vicarious learning: A review of the literature. Nurse Education in Practice 10(1), 13-16. doi: 0.1016/j.nepr.2009.01.017

Salfi, J., \& Carbol, B. (2017). The applicability of the NCLEX-RN to the Canadian testing population: A review of regulatory body evidence. International Journal of Nursing Education Scholarship, 14(1). doi:10.1515/ijnes-2016-0078

Sanford, J., Townsend-Rocchiccioli, J., Quiett, K., \& Trimm, D. (2011). "I see my mother' s face": Student nurse experiences caring for cancer patients. European Journal of Oncology Nursing, 15(1), 46-52. doi:10.1016/j.ejon.2010.05.010

Sellman, D. (2016). From CRNE to NCLEX-RN: Musings on nursing and the idea of a national final examination. Nursing Philosophy, 17(4), 227-228. doi:10.1111/nup.12145

Suliman, W.A., \& Halabi, J. (2007). Critical thinking, self-esteem, and state anxiety of nursing students. Nursing Education Today, 27, 162 168. doi:10.1016/j.nedt.2006.04.008

Tanner, C.A. (2006). Thinking like a nurse: A research-based model of clinical judgment in nursing. Journal of Nursing Education, 45, 204-211.

van Manen, M. (1990). Researching lived experience: Human science for an action sensitive pedagogy. Albany, NY: State University of New York Press.

Wilson, J.E.H., Azzopardi, W., Sager, S., Gould, B., Conroy, S., Deegan, P., \& Archie, S., (2009). A narrative study of the experiences of student nurses who have participated in the Hearing Voices that are Distressing simulation. International Journal of Nursing Education Scholarship, 6(1), 1-15. doi:10.2202/1548-923X.1727

Wong, F.K., \& Lee, W.M. (2000). A phenomenological study of early nursing experiences in Hong Kong. Journal of Advanced Nursing, 31(6), 1509-1517.

Wotton, K., Davis, J., Button, D., \& Kelton, M. (2010). Third-year undergraduate nursing students' perceptions of high-fidelity simulation. Journal of Nursing Education, 49(11), 632-639.

Wyatt, D.E. (2007). The impact of oncology education on practice-A literature review. European Journal of Oncology Nursing, 11, 255261. doi:10.1016/j.ejon.2006.09.002

Yildiz, H., \& Akansel, N. (2011). Beginning level nursing students' experiences with cancer patients in their first clinical placement: A qualitative appraisal in Turkey. Asian Pacific Journal of Cancer Prevention, 12(10), 2611-2615. 